International Journal of Modern Physics: Conference Series

(C) World Scientific Publishing Company

\title{
Are there infinitely many decompositions of the nucleon spin ?
}

\author{
Masashi Wakamatsu \\ Department of Physics, Faculty of Science, \\ Osaka University, Toyonaka, Osaka 560-0043, Japan \\ Received Day Month Year \\ Revised Day Month Year
}

\begin{abstract}
We argue against the rapidly spreading idea of gauge-invariant-extension (GIE) approach in the nucleon spin decomposition problem, which implies the existence of infinitely many gauge-invariant decomposition of the nucleon spin.

Keywords: nucleon spin decomposition; gauge-invariance; gluon spin evolution.

PACS numbers: 12.38.-t, 12.20.-m, 14.20.Dh, 03.50.De
\end{abstract}

\section{Introduction}

We now believe that only $1 / 3$ of the nucleon spin comes from the intrinsic spin of quarks. What carries the remaining $2 / 3$ of the nucleon spin, then ? This is one of the fundamental questions of QCD. To answer this question unambiguously, we must first clarify the following issues. What is a precise definition of each term of the decomposition in QCD ? How can we extract individual term by means of direct measurements ? Let us call it the nucleon spin decomposition problem. Since QCD is a color $\mathrm{SU}(3)$ gauge theory, the color gauge-invariance plays a crucial role in this problem. The reason is because the general gauge-invariance is a necessary condition of observability. Unfortunately, it is a very delicate problem, which is still under debate. The conflict appears to lie in the interpretation of the idea of gauge-invariance.

\section{Nucleon spin decomposition problem and its status}

It has been long known that there are two popular decompositions of the nucleon spin. One is the Jaffe-Manohar decomposition 1, and the other is the Ji decomposition 2. In these two decompositions, only the intrinsic quark spin part is common and the other parts are all different. A disadvantage of the Jaffe-Manohar decomposition is that each term is not separately gauge-invariant except for the quark spin part. On the other hand, each term of the Ji decomposition is separately gaugeinvariant. Unfortunately, it was claimed and has been widely believed that further gauge-invariant decomposition of $J^{g}$ into its spin and orbital parts is impossible. 
Several years ago, however, Chen et al. proposed a new gauge-invariant complete decomposition of the nucleon spin 3 . The basic idea is a decomposition of the total gluon field into the physical and pure-gauge components as $\boldsymbol{A}(x)=\boldsymbol{A}_{\text {phys }}(x)+$ $\boldsymbol{A}_{\text {pure }}(x)$, which is a sort of generalization of the decomposition of photon field in QED into the transverse $\boldsymbol{A}_{\perp}$ and longitudinal components $\boldsymbol{A}_{\|}$. Prominent features of their decomposition is that each term is separately gauge-invariant. It reduces to gauge-variant Jaffe-Manohar decomposition in a particular gauge $\boldsymbol{A}_{\text {pure }}=0, \boldsymbol{A}=$ $\boldsymbol{A}_{\text {phys }}$. Soon after, we have shown that the way of gauge-invariant decomposition of nucleon spin is not necessarily unique, and proposed another gauge-invariant decomposition 5 . The difference with the Chen decomposition appears in the orbital parts. The quark OAM part in our decompositin is the same as that of the Ji decomposition, while the gluon OAM part contain some extra term in addition to the canonical part. We call this piece the potential angular momenta term, because the QED correspondent of this term is the orbital angular momentum carried by the electromagnetic field or potential, appearing in the famous Feynman paradox of electrodynamics 6]. An arbitrariness of the spin decomposition arises, because this potential angular momentum term is solely gauge-invariant.

Furthermore, we found that we can make a seemingly covariant extension of the above two gauge-invariant decompositions of QCD angular momentum tensor, which we call the decompositions (I) and (II) 7 . The word "seemingly" is important here, because the decomposition $A^{\mu}(x)=A_{\text {phys }}^{\mu}(x)+A_{\text {pure }}^{\mu}(x)$, which is a foundation of the above gauge-invariant decompositions, is intrinsically non-covariant or frame-dependent, as we shall see. Still, this generalization is useful to find relations to high-energy DIS observables 879. Moreover, we pointed out that the decomposition (II) reduces to any ones of Bashinsky-Jaffe, of Chen et al., and of Jaffe-Manohar, after an appropriate gauge-fixing in a suitable Lorentz frame. Since the quark and gluon OAMs in these decompositions are physically the canonical orbital angular momenta, they may be called the "caninical" family. On the other hand, our decomposition (I) is an extension of the Ji decomposition in the sense that gluon part can also be gauge-invariantly decomposed into the orbital and intrinsic spin part. The quark OAM part in this decomposition contains the full covariant derivative, while the gluon part contains the seemingly covariant generalization of the potential OAM as well. Our central claim is that these two decompositions (I) and (II) basically exhaust possible gauge-invariant decompositions of the nucleon spin. However, an opposing claim has rapidly spread in the community 10|11/12. The claim is that, since the decomposition of the gauge field into its physical and pure-gauge components is not unique and there are infinitely many such decompositions, there are in principle infinitely many GI decompositions of the nucleon spin.

An argument in favor of the second claim was developed by Ji et al. 10. According to them, the Chen decomposition is a gauge-invariant extension (GIE) of the Jaffe-Manohar decomposition based on the Coulomb gauge, while the BashinskyJaffe decomposition is a GIE of the Jaffe-Manohar decomposition based on the light-cone gauge. Because the way of GIE with use of a path-dependent Wilson line 
is not unique, there is no need that the two decompositions give the same physical predictions. One should recognize the oblique nature of the word "GIE". In fact, assume that the Chen decomposition and the Bashinsky-Jaffe decompositions are two physically inequivalent GIEs of the Jaffe-Manohar decomposition. This immediately raises following questions. What is the physical meaning of extended gauge symmetries ? Are there plural color gauge symmetries in nature ? Our viewpoint, which we believe is standard, is that the color gauge symmetry is an intrinsic property of $\mathrm{QCD}$, which is present from the beginning and in principle there is no need of extending it. The gauge symmetry is rather freedoms to be eliminated by gauge-fixing procedures rather than to be obtained by extension.

Another argument in favor of the existence of infinitely many decompositions of the nucleon spin was developed by Lorcé 1112 . According to him, the Chen decomposition is a GIE based on the Stückelberg trick. There is a hidden symmetry called the Stückelberg symmetry, under which the pure-gauge and physical components respectively transform as follows :

$$
\begin{aligned}
& A_{\mu}^{\text {pure }}(x) \rightarrow A_{\mu}^{\text {pure }}(x)+\frac{i}{g} U_{\text {pure }}(x) U_{0}^{-1}(x)\left[\partial_{\mu} U_{0}(x)\right] U_{\text {pure }}^{-1}(x), \\
& A_{\mu}^{\text {phys }}(x) \rightarrow A_{\mu}^{\text {phys }}(x)-\frac{i}{g} U_{\text {pure }}(x) U_{0}^{-1}(x)\left[\partial_{\mu} U_{0}(x)\right] U_{\text {pure }}^{-1}(x) .
\end{aligned}
$$

Since this transformation leaves the total gluon field unchanged, there can be infinitely many decompositions of $A_{\mu}(x)$ into physical and pure-gauge components and consequently infinitely many decompositions of the nucleon spin. We claim and in fact showed that, in the QED case, the Chen decomposition is not a GIE based on the Stückelberg trick. (See sect. III of 9 and the whole discussion in 13.)

\section{Chen decomposition is not a GIE a la Stückelberg}

As is well-known, the vector potential of the photon field can be decomposed into transverse and longitudinal components as $\boldsymbol{A}=\boldsymbol{A}_{\perp}+\boldsymbol{A}_{\|}$, satisfying the divergence-free and irrotational conditions, $\nabla \cdot \boldsymbol{A}_{\perp}=0, \nabla \times \boldsymbol{A}_{\|}=0$. This transverselongitudinal decomposition is known to be unique, once the Lorentz frame of reference is fixed 14. Under a general gauge-transformation given as

$$
A^{0}(x) \rightarrow A^{\prime 0}(x)=A^{0}(x)-\frac{\partial}{\partial t} \omega(x), \quad \boldsymbol{A}(x) \rightarrow \boldsymbol{A}^{\prime}(x)=\boldsymbol{A}(x)+\nabla \omega(x),
$$

the transverse and longitudinal components transform as follows,

$$
\boldsymbol{A}_{\perp}(x) \rightarrow \boldsymbol{A}_{\perp}^{\prime}(x)=\boldsymbol{A}_{\perp}(x), \quad \boldsymbol{A}_{\|}(x) \rightarrow \boldsymbol{A}_{\|}^{\prime}(x)=\boldsymbol{A}_{\|}(x)+\nabla \omega(x),
$$

which means that the longitudinal component carries unphysical gauge degrees of freedom, while the transverse part is gauge-invariant.

Naturally, the longitudinal-transverse decomposition of the 3 -vector potential is Lorentz-frame dependent. (Anyhow, the whole treatment above is non-covariant.) It is true that a vector field that appears transverse in a certain Lorentz frame 
is not necessarily transverse in another Lorentz frame. Nonetheless, the Lorentzframe dependence of the transverse-longitudinal decomposition should not make any trouble, because one can start this decomposition in an arbitrarily chosen Lorentz frame. After all, the gauge- and frame-independence of observables is the core of the celebrated Maxwell's electrodynamics as a Lorentz-invariant gauge theory.

This QED example indicates that, as long as we are working in a chosen Lorentz frame, there is no arbitrariness in the decomposition of $A^{\mu}$, as arising from the Stückelberg-like transformation of Lorcé $11 \mid 12$. In fact, first note that the Stückelberg transformation in the abelian case reduced to the following simple form :

$$
\begin{aligned}
& A_{\mu}^{\text {pure }}(x) \rightarrow A_{\mu}^{\text {pure }, g}(x)=A_{\mu}^{\text {pure }}(x)-\partial_{\mu} C(x), \\
& A_{\mu}^{\text {phys }}(x) \rightarrow A_{\mu}^{\text {phys }, g}(x)=A_{\mu}^{\text {phys }}(x)+\partial_{\mu} C(x),
\end{aligned}
$$

with $C(x)$ being an arbitrary function of space-time. This certainly does not change the sum of the physical and pure-gauge components, Under this Stückelberg, however, the longitudinal and transverse components transform as follows :

$$
\begin{aligned}
& \boldsymbol{A}_{\|}(x) \rightarrow \boldsymbol{A}_{\|}^{g}(x)=\boldsymbol{A}_{\|}(x)-\nabla C(x), \\
& \boldsymbol{A}_{\perp}(x) \rightarrow \boldsymbol{A}_{\perp}^{g}(x)=\boldsymbol{A}_{\perp}(x)+\nabla C(x) .
\end{aligned}
$$

One sees that this transformation leaves the irrotational property of the longitudinal component unchanged :

$$
\nabla \times \boldsymbol{A}_{\|}^{g}(x)=\nabla \times\left(\boldsymbol{A}_{\|}(x)-\nabla C(x)\right)=\nabla \times \boldsymbol{A}_{\|}(x) .
$$

However, the divergence-free or the transversity condition is not preserved by this transformation,

$$
\nabla \cdot \boldsymbol{A}_{\perp}^{g}(x)=\nabla \cdot\left(\boldsymbol{A}_{\perp}(x)+\nabla C(x)\right)=\nabla \cdot \boldsymbol{A}_{\perp}(x)+\Delta C(x) \neq \nabla \cdot \boldsymbol{A}_{\perp}(x),
$$

unless the function $C(x)$ satisfies the 3-dimensional Laplace equation. This means that we can take $C(x)=0$ without loss of generality, so that there is no arbitrariness of Stückelberg transformation. The fact is that, while the pure-gauge part changes arbitrarily under the gauge-transformation, the physical part is essentially a unique object, constrained by the transversality condition.

\section{What is needed to settle the controversies}

What is needed to settle the controversies ? We recall that the main criticism from the GIE approach with use of the Wilson-line is that the decomposition $A_{\mu}(x)=A_{\mu}^{\text {phys }}(x)+A_{\mu}^{\text {pure }}(x)$ is not unique at all, i.e. there are infinitely many such decompositions arising from infinitely many choices of paths. From a physical viewpoint, however, the massless gauge field has only two physical or transverse degrees of freedom, and other components are unphysical gauge degrees of freedom. The standard gauge-fixing procedure is essentially the process of projecting out the two transverse or physical components of gauge field. Corresponding to the fact that 
there exist many gauge-fixing procedures, the expression of the physical component is not naturally unique. Nevertheless, an important wisdom is that final physical predictions for gauge invariant quantities are independent of the choice of gauges !

To reveal a hidden problem of the GIE approach, we briefly overview DeWitt's gauge-invariant formulation of QED 15 . For a given set of electron and photon fields, he constructed a gauge-invariant set of those in the following manner :

$$
\psi^{\prime}(x) \equiv e^{i \Lambda(x)} \psi(x), \quad A_{\mu}^{\prime}(x) \equiv A_{\mu}(x)+\partial_{\mu} \Lambda(x)
$$

with

$$
\Lambda(x)=-\int_{-\infty}^{0} A_{\sigma}(z) \frac{\partial z^{\sigma}}{\partial \xi} d \xi
$$

where $z^{\mu}(x, \xi)$ stands for a path satisfying the following boundary condition :

$$
z^{\mu}(x, 0)=x^{\mu}, \quad z^{\mu}(x,-\infty)=\text { spatial infinity }
$$

The problem is that, while $\psi^{\prime}(x)$ and $A_{\mu}^{\prime}(x)$ are gauge-invariant by construction, they are generally path-dependent.

The path-dependence can easily be understood by considering the simplest case of constant-time paths, which amounts to taking the following $\Lambda(x)$,

$$
\Lambda(x)=-\int_{-\infty}^{x} \boldsymbol{A}\left(x^{0}, \boldsymbol{z}\right) \cdot d \boldsymbol{z}
$$

Let us introduce two GI electron fields corresponding to two different choices of paths $L_{1}$ and $L_{2}$ :

$$
\begin{aligned}
& \psi^{\prime}\left(x ; L_{1}\right)=\exp \left[-i e \int_{L_{1}}^{x} \boldsymbol{A}\left(x^{0}, \boldsymbol{z}\right) \cdot d \boldsymbol{z}\right] \psi(x), \\
& \psi^{\prime}\left(x ; L_{2}\right)=\exp \left[-i e \int_{L_{2}}^{x} \boldsymbol{A}\left(x^{0}, \boldsymbol{z}\right) \cdot d \boldsymbol{z}\right] \psi(x) .
\end{aligned}
$$

The relation between these two electron fields is given by

$$
\psi^{\prime}\left(x ; L_{1}\right)=\exp \left[i e\left(\int_{L_{1}}^{x}-\int_{L_{2}}^{x}\right) \boldsymbol{A}\left(x^{0}, \boldsymbol{z}\right)\right] \psi^{\prime}\left(x ; L_{2}\right) .
$$

Closing the path to a loop $L$ by a connection at spatial infinity, we get the following relation :

$$
\begin{aligned}
\psi^{\prime}\left(x ; L_{1}\right) & =\exp \left[i e \oint_{L} \boldsymbol{A}\left(x^{0}, \boldsymbol{z}\right) \cdot d \boldsymbol{z}\right] \psi^{\prime}\left(x ; L_{2}\right) \\
& =\exp \left[i e \iint_{S}\left(\nabla_{z} \times \boldsymbol{A}\left(x^{0}, \boldsymbol{z}\right)\right) \cdot d \boldsymbol{z}\right] \psi^{\prime}\left(x ; L_{2}\right) \\
& =\exp \left[i e \iint_{S} \boldsymbol{B}\left(x^{0}, \boldsymbol{z}\right) \cdot d \boldsymbol{z}\right] \psi^{\prime}\left(x ; L_{2}\right)
\end{aligned}
$$

Here, we have used the Stokes theorem. Since the magnetic flux does not vanish in general, $\psi^{\prime}(x)$ is generally path-dependent. 
Why is the path-dependence a problem ? This is because the past researches clearly show that the path-dependence is a reflection of the gauge-dependence 16/17 1819. However, there are some nontrivial choices of the function $\Lambda(x)$, which leads to path-independent set of electron and photon fields, The first example is given by the choice 20 ,

$$
\Lambda(x)=-\int_{-\infty}^{x} \boldsymbol{A}_{\|}\left(x^{0}, \boldsymbol{z}\right) \cdot d \boldsymbol{z} .
$$

Here, $\boldsymbol{A}_{\|}(x)$ is the the longitudinal component of the photon. Since the closed-loop line integral of $\boldsymbol{A}_{\|}(x)$ vanishes as

$$
\oint_{L} \boldsymbol{A}_{\|}\left(x^{0}, \boldsymbol{z}\right) \cdot d \boldsymbol{z}=\iint_{S}\left(\nabla_{z} \times \boldsymbol{A}_{\|}\left(x^{0}, \boldsymbol{z}\right)\right) \cdot d \boldsymbol{S}=0,
$$

due to the property $\nabla \times \boldsymbol{A}_{\|}=0$, the electron field $\psi^{\prime}(x)$ defined with the above $\Lambda(x)$ is not only gauge-invariant but also path-independent !

If one remembers the familiar formula for the longitudinal component,

$$
\boldsymbol{A}_{\|}(x)=\nabla \frac{1}{\nabla^{2}} \nabla \cdot \boldsymbol{A}(x), \quad \boldsymbol{A}_{\perp}(x)=\boldsymbol{A}(x)-\boldsymbol{A}_{\|}(x),
$$

one can also express as follows :

$$
\begin{aligned}
\psi^{\prime}(x) & =\exp \left[-e \int_{-\infty}^{x}\left(\nabla_{z} \frac{1}{\nabla_{z}^{2}} \nabla_{z} \cdot \boldsymbol{A}\left(x^{0}, \boldsymbol{z}\right)\right) \cdot d \boldsymbol{z}\right] \psi(x) \\
& =\exp \left[-i e \frac{\nabla \cdot \boldsymbol{A}}{\nabla^{2}}(x)\right] \psi(x) .
\end{aligned}
$$

In this form, the path-independence of the GI electron field is self-evident. Note that this $\psi^{\prime}(x)$ is nothing but the GI physical electron introduced by Dirac 21/22. Using the same function $\Lambda(x)$, the GI potential $A_{\mu}^{\prime}(x)$ becomes

$$
\boldsymbol{A}^{\prime}(x)=\boldsymbol{A}_{\perp}(x), \quad A^{\prime 0}(x)=A^{0}(x)+\int_{-\infty}^{x} \boldsymbol{A}_{\|}\left(x^{0}, \boldsymbol{z}\right) \cdot d \boldsymbol{z} .
$$

One thus reconfirms that the physical component of the spatial part of the photon field is nothing but the familiar transverse component.

Also interesting is the following second example. Using a constant 4 -vector $n^{\mu}$, we introduce the decomposition :

$$
A_{\mu}(x)=A_{\mu}^{\text {phys }}(x)+A_{\mu}^{\text {pure }}(x) \equiv\left(P_{\mu \nu}+Q_{\mu \nu}\right) A^{\nu}(x),
$$

with

$$
P_{\mu \nu}=g_{\mu \nu}-\frac{\partial_{\mu} n_{\nu}}{n \cdot \partial}, \quad Q_{\mu \nu}=\frac{\partial_{\mu} n_{\nu}}{n \cdot \partial}
$$

These two components satisfy the important properties :

$$
n^{\mu} A_{\mu}^{\text {phys }}(x)=0, \quad \partial_{\mu} A_{\nu}^{\text {pure }}(x)-\partial_{\nu} A_{\mu}^{\text {pure }}=0 .
$$


Now, we propose to take the following $\Lambda(x)$,

$$
\Lambda(x)=-\int_{-\infty}^{x} A_{\mu}^{\text {pure }}(z) d z^{\mu},
$$

and define the GI electron and photon fields by Eq.(11). Note that, by using the Stokes theorem in 4 space-time dimension, the following identity holds

$$
\oint_{L} A_{\mu}^{\text {pure }}(z) d z^{\mu}=\frac{1}{2} \iint_{S}\left(\partial_{\mu} A_{\nu}^{\text {pure }}-\partial_{\nu} A_{\mu}^{\text {pure }}\right) d \sigma^{\mu \nu}=0,
$$

so that this $\Lambda(x)$ turns out to be path-independent. In fact, $\Lambda(x)$ can also be expressed in the following form :

$$
\Lambda(x)=-\int_{-\infty}^{x} \frac{\partial_{\mu}^{z} n_{\nu}}{n \cdot \partial^{z}} A^{\nu}(z) d z^{\mu}=\frac{n \cdot A(x)}{n \cdot \partial} .
$$

The GI electron and photon fields are then given by

$$
\psi^{\prime}(x)=e^{i e \frac{n \cdot A(x)}{n \cdot \partial}} \psi(x), \quad A_{\mu}^{\prime}(x)=\left(g_{\mu \nu}-\frac{\partial_{\mu} n_{\nu}}{n \cdot \partial}\right) A^{\nu}(x)=A_{\mu}^{p h y s}(x) .
$$

Note that the physical component satisfies the following condition,

$$
n^{\mu} A_{\mu}^{\text {phys }}(x)=0
$$

which is nothing but the gauge-fixing condition in general axial gauge.

These two examples clearly show that the form of the physical component is not in fact unique. It is expressed in several different forms, which is not unrelated to the fact that there are many gauge-fixing procedures in different Lorentz frame. Nevertheless, standard belief is that, as far as we handle the gauge- and Lorentzinvariant quantity in a usual sense, the final prediction should be the same. After this pedagogical introduction, we now want to address our central question. Is the gluon spin term appearing in the longitudinal nucleon spin sum rule such a quantity with standard gauge-invariance or not? To answer this question, we must generalize the construction of $A_{\mu}^{\text {phys }}(x)$ to the nonabelian gauge theory. We point out that, in the past, tremendous efforts have been made to figure out the two physical components of the gauge field.

Especially useful for our purpose is the geometrical construction by Ivanov, Korchemsky, and Radyushkin based on the fiber-bundle formulation of gauge theories 23. In their formulation, the gauge-covariant gluon field can be constructed in the following form :

$$
A_{\mu}^{g}(x)=A_{\nu}\left(x_{0}\right) \frac{\partial x_{0}^{\nu}}{\partial x^{\mu}}-\int_{x_{0}}^{x} d z^{\nu} \frac{\partial z^{\rho}}{\partial x^{\mu}} W_{C}\left(x_{0}, z\right) F_{\nu \rho}(z ; A) W_{C}\left(z, x_{0}\right),
$$

where

$$
W_{C}\left(x, x_{0}\right) \equiv P \exp \left[i g \int_{x_{0}}^{x} d z^{\mu} A_{\mu}(z)\right]
$$

is a familiar Wilson line with $z(s)$ being a path $C$ in 4-dimensional space-time with an appropriate boundary condition. One should clearly keep in mind the fact that 
$A_{\mu}^{g}(x)$ so constructed is generally dependent of the choice of path $C$. However, these authors clearly recognize the fact that the choice of suitable path in the geometrical formulation corresponds to gauge-fixing procedure. They also showed that, with some natural choices of paths, the above way of fixing the gauge is equivalent to taking gauges satisfying a particularly simple condition $W_{C}\left(x, x_{0}\right)=1$. This class of gauge is called the contour gauge and it is shown to have an attractive feature that they are ghost-free. Some familiar gauges belonging to the contour gauge are the Fock-Schwinger gauge, the Hamilton gauge, and the axial gauge. In particular, the axial gauge corresponds to taking an infinitely long straight-line path $z^{\mu}(s)=x^{\mu}+s n^{\mu}(0<s<\infty)$. This gives the following expression for $A_{\mu}^{g}(x)$ :

$$
A_{\mu}^{g}(x)=n^{\nu} \int_{0}^{\infty} W_{C}^{\dagger}(x+n s, \infty) F_{\mu \nu}(x+n s ; A) W_{C}(x+n s, \infty),
$$

with

$$
W_{C}(x, \infty)=P \exp \left(i g \int_{0}^{\infty} d s n^{\mu} A_{\mu}(x+n s)\right) .
$$

Using the antisymmetry of the field-strength tensor, it is easy to verify the identity $n^{\mu} A_{\mu}^{g}=0$, which is nothing but the gauge-fixing condition in general axial gauge. Since $n^{\mu}$ is an arbitrary constant 4 -vector, it contains several popular gauges, i.e. the temporal gauge, the light-cone gauge, and the spatial axial-gauge, respectively corresponding to the choice $n^{\mu}=(1,0,0,0), n^{\mu}=(1,0,0,1) / \sqrt{2}$ and $n^{\mu}=(0,0,0,1)$.

Since our main interest here is to show the traditional gauge-invariance of the evolution equation of the longitudinal gluon spin, let us inspect the perturbative (lowest order) contents of the defining equation of the physical component $A_{\mu}^{\text {phys }}(x) \equiv A_{\mu}^{g}(x)$, which reduces to

$$
A_{\mu}^{\text {phys }}(x) \simeq n^{\nu} \int_{0}^{\infty} d s\left(\partial_{\mu} A_{\nu}(x+n s)-\partial_{\nu} A_{\mu}(x+n s)\right) .
$$

Introducing the Fourier transform, this physical component can be expressed as,

$$
\begin{aligned}
A_{\mu}^{p h y s}(x) & \simeq n^{\nu} \int_{0}^{\infty} d s \int \frac{d^{4} k}{(2 \pi)^{4}} e^{i k \cdot(x+n s)}\left(i k_{\mu} \tilde{A}_{\nu}(k)-i k_{\nu} \tilde{A}_{\mu}(k)\right) \\
& =\int \frac{d^{4} k}{(2 \pi)^{4}}\left(g_{\mu \nu}-\frac{k_{\mu} n_{\nu}}{k \cdot n}\right) \tilde{A}^{\nu}(k)=\left(g_{\mu \nu}-\frac{\partial_{\mu} n_{\nu}}{n \cdot \partial}\right) A^{\nu}(x) .
\end{aligned}
$$

(Note that, this is an exact expression in the abelian case.) This in turn gives the lowest order expression for the physical gluon propagator as follows,

$$
\left\langle T\left(A_{\mu, a}^{p h y s}(x) A_{\nu, b}^{p h y s}(y)\right)\right\rangle^{(0)}=\int \frac{d^{4} k}{(2 \pi)^{4}} e^{i k(x-y)} \frac{-i \delta_{a b}}{k^{2}+i \varepsilon} P_{\mu \nu}(k),
$$

with

$$
P_{\mu \nu}(k)=g_{\mu \nu}-\frac{k_{\mu} n_{\nu}+n_{\mu} k_{\nu}}{k \cdot n}+\frac{n^{2} k_{\mu} k_{\nu}}{(k \cdot n)^{2}},
$$

which is nothing but the gluon propagator in the general axial gauge. In this way, the path dependence or direction dependence in the geometric formulation is replaced 
by the gauge dependence within the general axial gauge. In this setting, the gluon spin operator reduces to the form :

$$
M_{G-s p i n}^{\lambda \mu \nu}=2 \operatorname{Tr}\left[F^{\lambda \nu} A^{\mu}-F^{\lambda \mu} A^{\nu}\right] .
$$

In this equation, $A^{\mu}$ should be regarded as the physical gluon field satisfying the general axial gauge condition.

\section{Evolution equation for the gluon spin in general axial gauge}

Now, we are ready to investigate the evolution equation for the quark and gluon spins in general axial gauge. Let us start with the following covariant relation,

$$
\left\langle P s\left|M^{\lambda \mu \nu}(0)\right| P s\right\rangle=J_{N} \frac{P_{\rho} s_{\sigma}}{M_{N}^{2}}\left[2 P^{\lambda} \epsilon^{\nu \mu \rho \sigma}-P^{\mu} \epsilon^{\lambda \nu \rho \sigma}-P^{\nu} \epsilon^{\mu \lambda \rho \sigma}\right] .
$$

The longitudinal nucleon spin sum rule is obtained by setting $\mu=1, \nu=2$, and by contracting with the constant 4 -vector $n^{\mu}$, which gives

$$
J_{N}=\frac{1}{2}=\frac{\left\langle P s\left|n_{\lambda} M^{\lambda 12}(0)\right| P s\right\rangle}{2 P \cdot n} .
$$

An important fact is that this last equation is no longer a covariant relation. The quantity $n_{\lambda}$ appearing in this equation should be identified with the 4 -vector that characterizes the Lorentz-frame, in which the gauge-fixing condition $n^{\mu} A_{\mu}=0$ is imposed. In this setting, we have calculated the 1-loop anomalous dimension of the above gluon spin operator, and found that it reproduces the commonly-known answer, irrespectively of the choice of $n^{\mu}$. Although this is a proof within a restricted class of gauge, i.e. the general axial gauge, characterized by a constant 4 -vector $n^{\mu}$, it strongly indicates that the gluon spin term in the longitudinal nucleon spin sum rule is a gauge-invariant quantity in a true or traditional sense. This is a welcome conclusion, because it means that now there is no conceptual conflict between the observability of the nucleon spin decomposition (I) and the general gauge principle.

\section{Conclusion}

We have carried out a detailed comparison of the two fundamentally different approaches to the nucleon spin decomposition problems, i.e. the GIE approach and the standard gauge-fixing approach. If both give the same answer, there is no practical problem. However, if they give different answers, one must stop and think it over. In our opinion, conceptually legitimate is the latter approach. For, there is only one color gauge symmetry of QCD, which is present from the beginning. This gauge symmetry is rather freedoms to be eliminated by gauge-fixing procedures rather than to be gained by extension. This general consideration gives a support to our claim that there are only two (not infinitely many) physically inequivalent GI decompositions (I) and (II) of the nucleon spin. 


\section{Acknowledgments}

The author would like to greatly appreciate many stimulating discussions with Cedric Lorcé, and also with Takahiro Kubota.

\section{References}

1. R.L. Jaffe and A. Manohar, Nucl. Phys. B337, 509 (1990).

2. X. Ji, Phys. Rev. Lett. 78, 610 (1997).

3. X.S. Chen, X.F. Lü, W.M. Sun, F. Wang, and T. Goldman, Phys. Rev. Lett. 100, $232002(2008)$.

4. X.S. Chen, W.M. Sun, X.F. Lü, F. Wang, and T. Goldman, Phys. Rev. Lett. 103, 0620011 (2009).

5. M. Wakamatsu, Phys. Rev. D81, 114010 (2010).

6. R.P. Feynman, R.B. Leighton, and M.L. Sands, Feynman Lectures on Physics, Vol.III (Addison-Wesley, Reading, MA, 1965).

7. M. Wakamatsu, Phys. Rev. D83, 014012, (2011).

8. M. Wakamatsu, Phys. Rev. D84, 037501, (2011).

9. M. Wakamatsu, Phys. Rev. D85, 114039, (2012).

10. X. Ji, Y. Xu, and Y. Zhao, JHEP 1208, 082 (2012).

11. C. Lorcé, Phys. Rev. D87, 034031 (2013).

12. C. Lorcé, Phys. Lett. B719, 185 (2013).

13. M. Wakamatsu, Phys. Rev. D87, 094035 (2013).

14. C. Cohen-Tanouji, J. Dupont-Roc, and G. Grynberg, Photon \& Atoms (Wiley, New York, 1989).

15. B.S. DeWitt, Phys. Rev. 125, 2189 (1962).

16. F. Belinfante, Phys. Rev. D128, 2832 (1962).

17. S. Mandelstam, Ann. Phys. 19, 1 (1962).

18. F. Rohrlich F and F. Strocchi, Phys. Rev. 139, B476 (1965).

19. K.-H. Yang, J. Phys. A : Math Gen. 18, 979 (1985).

20. T. Kashiwa and Y. Takahashi, arXiv : hep-th/9401097(1994).

21. P.M. Dirac, Principles of Quantum Mechanics (Oxford University, New York, 1958).

22. M. Lavelle and D. McMullan, Phys. Lett. B312, 211 (1993).

23. S.V. Ivanov, G.P. Korchemsky, and A. Radyushkin, Yad. Fiz. 44, 230 (1986) [Sov. J. Nucl. Phys. 44, 145 (1986)]. 